\title{
An Interstitial Network of Podoplanin-Expressing Cells in the Human Endolymphatic Duct
}

\author{
Anna-Karin Hultgård-Ekwall, ${ }^{1,2}$ Christina Mayerl, ${ }^{3}$ Kristofer Rubin, ${ }^{1}$ Georg Wick, ${ }^{3}$ \\ AND Helge Rask-ANDERSEN ${ }^{2}$ \\ ${ }^{1}$ Department of Medical Biochemistry and Microbiology, Uppsala University, BMC, Box 582, SE-751 23 Uppsala, Sweden \\ ${ }^{2}$ Department of Otosurgery, University Hospital, SE-751 85 Uppsala, Sweden \\ ${ }^{3}$ Division of Experimental Pathophysiology and Immunology, Biocenter, Innsbruck Medical University, Austria
}

Received: 7 June 2005; Accepted: 11 November 2005; Online publication: 12 January 2006

\begin{abstract}
The human endolymphatic duct (ED) with encompassing interstitial connective tissue (CT) is believed to be important for endolymph resorption and fluid pressure regulation of the inner ear. The periductal CT cells are interconnected via numerous cellular extensions, but do not form vessel structures. Here we report that the periductal CT is populated by two distinct cell phenotypes; one expressing podoplanin, a protein otherwise found on lymph endothelia and on epithelia involved in fluid fluxes, and a second expressing a fibroblast marker. A majority of the interstitial cells expressed podoplanin but not the lymphatic endothelial cell markers hyaluronan receptor (LYVE-1) or vascular endothelial growth factor receptor-3 (VEGFR-3). The fibroblast marker positive cells were found close to the ED epithelium. In the mid- and distal parts of the ED, these cells were enriched under folded epithelia. Furthermore, subepithelial CT cells were found to express activated platelet derived growth factor (PDGF)- $\beta$ receptors. Cultured CT cells from human inner ear periductal and perisaccular explant tissues were identified as fibroblasts. These cells compacted a three-dimensional collagen lattice by a process that could be promoted by PDGF-BB, a factor involved in interstitial fluid pressure regulation. Our results are com-
\end{abstract}

Correspondence to: Anna-Karin Hultgård-Ekwall • Department of Medical Biochemistry and Microbiology, Uppsala University, BMC, Box 582, SE-751 23 Uppsala, Sweden. Telephone: +46-18-4714933; Fax: +46-18-4714975; email: anna-karin.ekwall@imbim.uu.se patible with the notion that the periductal CT cells are involved in the regulation of inner ear fluid pressure. By active compaction of the periductal CT and by the formation of villous structures, the CT cells could modulate fluid fluxes over the ED epithelium as well as the longitudinal flow of endolymph in the ED.

Keywords: Inner ear, Endolymphatic duct, Loose connective tissue, Podoplanin, Ménière's disease

\section{INTRODUCTION}

The human endolymphatic duct (ED) is a nonsensory component of the endolymph-filled membranous labyrinth of the inner ear. The ED forms a narrow tubule with a single layer epithelium leading to the endolymphatic sac (ES) through the vestibular aqueduct (VA) of the petrous bone. The ED is surrounded by a loose interstitial connective tissue (CT) also filling up numerous bone cavities parallel to the aqueduct (Friberg et al. 1984). The interstitial cells are organized in a tissue network structure with prominent intercellular and cell-extracellular matrix (ECM) contacts (Hultgard-Ekwall et al. 2003). The CT network makes direct contact with the basal lamina of the ED epithelium and with the encompassing bone matrix.

The ED and ES are believed to be important for endolymph resorption and fluid pressure regulation in the inner ear (Rask-Andersen et al. 1999; Salt and 
DeMott 2000). Endolymph is thought to be produced in the stria vascularis of the cochlea and the dark cell region of the utricle, and under certain conditions to circulate through the labyrinth into the ED and ES. Although a few capillaries are present in the periductal CT, a major part of the fluid drainage is believed to occur through the vein(s) of the VA into intracranial sinuses. No lymph vessels have been detected in the human VA.

Podoplanin is a $43 \mathrm{kD}$ cell surface glycoprotein expressed on lymphatic but not blood endothelia (Kriehuber et al. 2001). Together with vascular endothelial growth factor receptor 3 (VEGFR-3) and the hyaluronan receptor LYVE-1, podoplanin constitute an important marker for lymphatic vessels, e.g. in tumors (Breiteneder-Geleff et al. 1999; Schoppmann et al. 2001). Podoplanin is also expressed on mesothelia, ependyma, choroid plexuses, ciliary epithelia and glomerular epithelia of the kidney. Podoplanin is homologous to the T1 $\alpha$ or E11 antigen, originally found selectively expressed on alveolar type I-cells in the rat lung (Rishi et al. 1995; Wetterwald et al. 1996; Williams et al. 1996). It is also homologous to the mouse glomerular membrane protein gp38P (Boucherot et al. 2002), the OTS-8 gene product in cultured mouse osteoblasts (Nose et al. 1990) and the $45 \mathrm{kD}$ protein PA2.26, originally identified as a cell-surface antigen induced in keratinocytes during mouse skin carcinogenesis and in dermal fibroblast-like cells during wound healing (Gandarillas et al. 1997). Although little is known about the biological functions of podoplanin, T1apodoplanin $-/-$ mice die at birth due to respiratory failure and have defects in lymphatic vessel formation (Schacht et al. 2003). Over-expression of T1apodoplanin/PA2.26 in cultured cells promotes the formation of cell-surface protrusions and increase adhesion and migration (Martin-Villar et al. 2005; Schacht et al. 2003). Podoplanin has also been suggested to play a role in maintaining the unique shape of the foot processes of podocytes (Breiteneder-Geleff et al. 1997; Matsui et al. 1999).

According to new concepts in CT physiology, fibroblasts of the CT control the interstitial fluid pressure and tissue volume via actively regulated intercellular adhesion and adhesion to fibers of the ECM, thereby restraining the intrinsic tendency of glycosaminoglycans to bind water and expand (Wiig et al. 2003). Platelet derived growth factor-BB (PDGF$\mathrm{BB}$ ) is a fibroblast mitogen and promigratory factor acting via tyrosine kinase (PDGF- $\alpha$ and $\beta$ ) receptor signaling to modulate different CT processes, e.g. wound healing (Heldin and Westermark 1999). PDGF-BB regulates interstitial fluid pressure in vivo (Rodt et al. 1996) and promotes fibroblast adhesion to ECM in vitro in collagen gel contraction experi- ments (Clark et al. 1989; Gullberg et al. 1990). We have inferred that interstitial fluid pressure regulation is important in the inner ear and especially for endolymph resorption.

The aim of this work was to seek the presence of interstitial fluid pressure regulating cells in the periductal CT and to investigate whether the tissue is likely to respond to $\mathrm{CT}$ modulating factors like PDGF-BB.

\section{MATERIALS AND METHODS}

\section{Specimens}

Ten human ED specimens were dissected out during skull base surgery on patients suffering from vestibular schwannoma, but otherwise with no known inner ear disease. The patient material equally represented sexes and age ranged from 41 to 73 years. Approval for these studies was obtained from the local medical ethics committee (no. 99398, 22/9 1999 and 29/12 2003). During the translabyrinthal procedure, the ED together with the intraosseous part of the ES and a small portion of bone was obtained by use of a diamond burr. Four ED specimens (ED104;106;108;110) were prepared for immunohistochemical studies; fixed in $4 \%$ paraformaldehyde (PFA) in phosphate buffered saline (PBS)-pH 7.2-7.4 for 8-12 h prior to decalcification for 14 days in $0.1 \mathrm{M}$ Na-EDTA. One ED specimen (ED103) was fixed in 3\% glutaraldehyde for transmission electron microscopy (TEM) studies. The use of directly fixed surgical material instead of postmortem material gives a greatly improved morphological preservation of the tissue.

\section{Explant cell cultures and cells}

The soft tissue from five ED specimens was separated from the bone, cut in smaller pieces and transferred to culture medium. The inner ear (IE) tissue pieces were placed in tissue culture dishes and grown in Dulbecco's Modified Eagle Medium (DMEM) with Glutamax, supplemented with penicillin-streptomycin and $10 \%$ fetal calf serum (FCS) (State Veterinary Institute, Uppsala, SE) in a humid chamber with $5.0 \% \mathrm{CO}_{2}$ and $37^{\circ} \mathrm{C}$. After $5-10$ days, cells grew out of the explant tissue to cover the culture dish. After four to five passages, cultures were homogenous with a majority of cells exhibiting a fibroblast phenotype. The IE cells were used up to passage eight. Human foreskin fibroblasts Ag 1518 (Genetic Mutant Cell Repository, Camden, NJ) were used before passage 20 and human dermal fibroblasts (primary explant cells) before passage five. 


\section{Transmission electron microscopy (TEM)}

TEM was performed as described by Hultgard-Ekwall et al. (2003). Briefly, the ED specimen was post-fixed in $1 \%$ osmium tetroxide, dehydrated in graded ethanols and embedded in Epon for thin sectioning. Sections were mounted on grids and stained with uranyl acetate and lead citrate, and viewed in a Jeol 100 SX electron microscope.

\section{Immunohistochemistry}

Six micrometer thick frozen sections of the ED specimens were dried over night, rehydrated, incubated for 20 min with a blocking buffer (Invitrogen) supplemented with $10 \%$ bovine calf serum, incubated with rabbit anti-human podoplanin antiserum (raised against purified recombinant podoplanin core protein, 1:1,000) or mouse anti-human fibroblast (clone TE-7, Cymbus, 1:100) or rabbit anti-human VEGFR-3 (Chemicon, 1:200) or rabbit anti-human LYVE-1 (Abcam, 1:100) antibodies or normal mouse/rabbit serum for $60 \mathrm{~min}$, rinsed and subsequently incubated with a labeled polymer conjugated goat anti-rabbit and mouse Ig-alkaline phosphatase (AP) (Dako, EnVision) or in two separate steps; biotinylated $\mathrm{F}\left(\mathrm{ab}^{\prime}\right)_{2}$ fragment of swine anti-rabbit- or rabbit antimouse Ig (Dako, 1:200) followed by streptavidin-AP (Vector, 1:200) for $30 \mathrm{~min}$. Fast Red (Sigma) was used as AP substrate. Counterstaining in Mayers hematoxylin (Merck, 1:40) or Mayers modified hematoxylin (Histolab; 1:20). Mounting in Kaiser's glycerol gelatin (Merck).

The PDGF- $\beta$ receptor staining was performed as described by Hultgard-Ekwall et al. (2003). In brief, mouse anti-human PDGFR-B2 $(10 \mu \mathrm{g} / \mathrm{ml})$ antibodies were detected using secondary biotinylated $F\left(a^{\prime}\right)_{2}$ fragment of rabbit anti-mouse antibodies (Dako, 1:200), horseradish peroxidase (HRP)-conjugated streptavidin and diaminobenzidine (DAB) substrate (Zymed).

\section{Immunocytochemistry}

Explant ED/ES cells or Ag 1518-fibroblasts were grown in eight-well chamber slides (Lab TEK) in DMEM-Glutamax with $10 \%$ fetal bovine serum. At about $50 \%$ confluence the cells were fixed in cold $4 \%$ PFA (10 min), permeabilized with $0.5 \%$ Triton $\mathrm{x}-100$ (15 min) and blocked with $0.1 \mathrm{M}$ Glycine- $0.5 \%$ BSA in PBS overnight at $+4^{\circ} \mathrm{C}$. Primary antibody incubation was performed with anti-human vimentin (clone 3B4, DAKO, 1:400), anti-human pan-cytokeratin (Sigma, 1:400), anti-human $\alpha$ and $\gamma$-actin (HHF 35, DAKO, 1:50), anti-human calponin (Sigma, 1:500), anti-human smooth muscle myosin (Sigma, 1:100), anti-human fibroblast clone 5B5 (DAKO, 1:100) or anti-human fibroblast clone TE-7 (Cymbus, 1:100) antibodies together with 5\% normal swine or rabbit serum (depending on secondary antibody species) for $60 \mathrm{~min}$. Secondary antibody- and substrate incubations, counter-staining and mounting were performed as described above under IH.

\section{Double immunofluorescence and confocal microscopy}

After incubation with blocking buffer, frozen sections from human ED and skin were subjected to rabbit anti-human podoplanin antibody $(1: 1,000)$ or control rabbit serum for $30 \mathrm{~min}$, rinsed, incubated with Alexa Fluor 488-conjugated goat anti-rabbit IgG (Molecular probes, 1:100) for $30 \mathrm{~min}$, rinsed, incubated with mouse anti-human fibroblast antibodies (clone TE-7, Cymbus, 1:100) or control mouse serum for $30 \mathrm{~min}$ and subsequently with, Alexa Fluor 568conjugated goat anti-mouse IgG (Molecular probes, 1:200) and DAPI nuclear staining (Sigma, 1:1,000) for $30 \mathrm{~min}$. Mounting in moviol and analysis in a Zeiss confocal microscope.

\section{Collagen gel contraction assay}

The assay was performed essentially as described by Gullberg et al. (1990). Explant ED/ES fibroblasts or Ag 1518-fibroblasts from confluent cultures were trypsinized, suspended in serum-free DMEM and diluted to 500,000 cells $/ \mathrm{ml}$. Each cell suspension was mixed on ice with a collagen type I solution ( $5 \mathrm{vol}$ 2xDMEM, 1 vol 0.2 M HEPES buffer $\mathrm{pH} 9.0$ and 4 vol Vitrogen 100 (Cohesion Technologies, 95-98\% collagen type $\mathrm{I}, 3 \mathrm{mg} / \mathrm{ml})$ ) at a $1: 9$ ratio. One hundred microliters of cell-collagen suspension were added per well to a BSA-coated 96-well microtiter plate. The plate was incubated at $37^{\circ} \mathrm{C}$ to allow for polymerization. After $11 / 2 \mathrm{~h}$, the gels were floated with $100 \mu \mathrm{l}$ DMEM containing $2 \times 1 \mu \mathrm{M}$ prostaglandin E1 (PGE1)-methyl ester (Sigma) or $2 \times 20 \mu \mathrm{g} / \mathrm{ml}$ platelet derived growth factor BB (PDGF-BB) or both (time=0). Collagen gel contraction was measured as decrease in gel area, using an inverted light microscope.

\section{RESULTS}

\section{Expression of podoplanin in the human ED}

Immunohistochemical staining of four ED tissue specimens showed positive expression for podoplanin in a majority of the periductal CT cells (Fig. 1A-D). A qualitatively similar staining pattern was obtained with all four specimens. Podoplanin positive cells were present both in the subepithelial stroma and in 

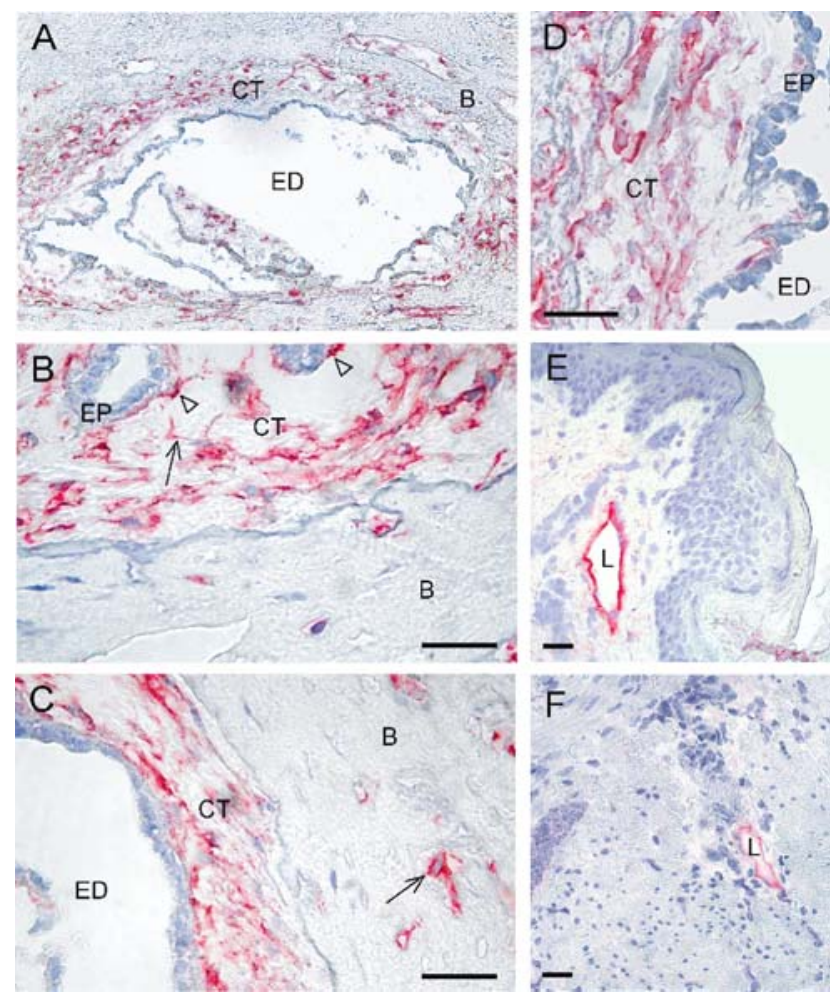

FIG. 1. Podoplanin-expression in human endolymphatic duct $(E D)$ specimens detected by immunohistochemical staining using an antipodoplanin antibody. Qualitatively similar staining was obtained with all four $E D$ specimens $(E D 104 ; 106 ; 108 ; 110)$. A The vestibular aqueduct with the $E D$ and encompassing interstitial $C T$. Podoplaninexpressing cells (red) in the $C T$ and in adjacent bone canaliculi. B Podoplanin-expressing cells in the distal part of the $E D /$ proximal part of the ES. Note the positive staining of long cellular extensions (arrow) and of cells juxta-positioned to the epithelium (arrow heads). C Podoplanin-expression in the proximal/mid part of the $E D$. Note the positive cells of the bone matrix (arrow). D Podoplaninexpressing cells in the mid part of the ED108-specimen. Note the lucid, fluid-rich subepithelial area. E A positive control staining of a lymph vessel on a human skin section, using the anti-podoplanin antibody. Note that the dermal fibroblasts are negative. $\mathbf{F}$ A positive control staining of a lymph vessel in the submucosa of a human large intestine specimen, using the anti-podoplanin antibody. Bar 50 $\mu \mathrm{m}$. $E D$ lumen of the endolymphatic duct, $C T$ interstitial connective tissue, $B$ bone, $E P$ epithelium, $L$ lymph vessel.

small cavities of the surrounding bone (Fig. 1A) and could be found at the epithelial-mesenchymal interface (Fig. 1B arrow heads). The podoplanin-expressing cells were detected in proximal, mid- and distal parts of the ED. Positive cells had a homogenous cytoplasmic staining pattern, present also in long cellular extensions (Fig. 1B, arrow). Podoplanin also stained osteoblasts/osteocytes in the adjacent bone tissue (Fig. 1C, arrow), whereas osteocytes in more distant bone tissue were negative (data not shown). The epithelial cells of the EDs were invariably negative. The anti-podoplanin antibody specifically stained lymphatic vessels but not dermal fibroblasts or any other CT cell in human skin and large intestine specimens (Fig. 1E and F).

\section{Expression of the Cymbus fibroblast marker} and PDGF- $\beta$ receptors in the ED

We have reported earlier that human periductal CT cells express prolyl-4-hydroxylase (collagen producing cells) and vimentin, but not $\alpha$-smooth muscle actin, desmin, CD31-blood endothelial marker, CD68-macrophage marker or cytokeratin (HultgardEkwall et al. 2003).

Here we show that a sub-population of the periductal CT cells, especially cells juxta-positioned to the epithelium, was stained positive with an antihuman fibroblast antibody from Cymbus (Fig. 2A). In agreement with earlier reports, the Cymbus antibody stained a majority of dermal fibroblasts in human skin specimens (Fig. 2B). Furthermore, subepithelial CT cells were found to express activated PDGF- $\beta$ receptors as detected by the monoclonal antibody PDGFR-B2 (Fig. 3A). Confocal image analysis of double stainings with PDGFR-B2 and Cymbus antibodies on ED specimens were not conclusive to whether the receptors were present on the epithelia or on the juxta-positioned fibroblasts (data not shown). TEM analysis of a human ED specimen verified the presence of CT cells juxta-positioned to the epithelium (Fig. 3B). These cells formed prominent contacts with the basal lamina, intercellular contacts and had long cellular extensions. In addition, TEM analysis indicated the presence of two heterogeneous periductal CT cell types. One concentrically arranged thin laminar cell with small nuclei and long thin cellular extensions (Fig. 3C, arrows), which preferentially was located close to the epithelium. At further distance from the ED lumen, large CT cells were found. This more abundant cell type had prominent nuclei, numerous organelle structures and short cytoplasmatic extensions forming frequent intercellular contacts (Fig. 3C, arrow heads). Based on locality, it is possible that the podoplanin-expressing cells are found in the latter population of cells.

\section{Expression of lymph endothelial markers} in the ED

Since podoplanin reportedly is present on lymph endothelial cells (Schoppmann et al. 2001), we investigated whether two other markers of lymph endothelia could be detected on the inner ear ED sections. Neither anti-LYVE-1 nor anti-VEGFR-3 antibody stained periductal CT cells or any other cells in the ED tissue specimens (Fig. 2C,E). In human large 


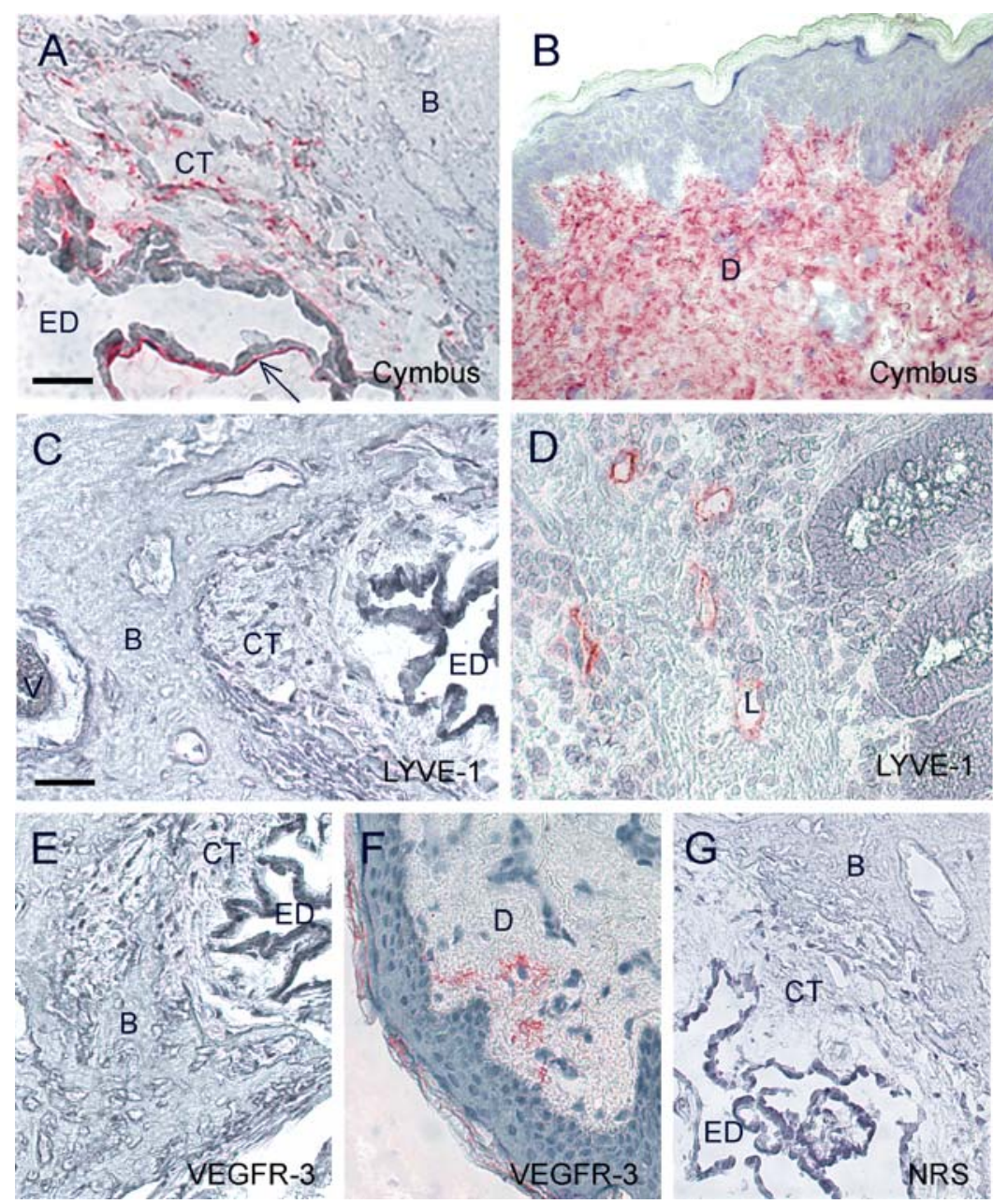

FIG. 2. Immunohistochemical staining of the human ED using the Cymbus anti-fibroblast antibody (A), staining $C T$ cells especially juxta-positioned to the epithelium (A, arrow), the lymphatic endothelial marker antibodies; anti-LYVE-1 (C) and anti-VEGFR-3 (E). Positive control staining of human skin specimens with the Cymbus antibody (B) and the anti-VEGFR-3 antibody (F), and human

intestine and skin specimens, LYVE-1 and VEGFR-3 specifically stained lymphatic vessels (Fig. 2D and F).

Podoplanin- and Cymbus fibroblast marker-expressing cells constituted two distinct cell populations in the ED

To further characterize the periductal CT cells, double immunofluorescence staining was performed. The anti-podoplanin and anti-fibroblast antibodies stained different cell populations in the periductal CT (Fig. 4A and B). In the proximal part of the ED (Fig. 4A), the fibroblast expressing cells were more abundant than in the mid- and distal parts (Fig. 4B). An overlapping staining was seen of CT cells juxta positioned to the epithelium in the proximal part of the ED. If this represented double-positive cells or juxta positioned cells could not be determined with large intestine specimen with the anti-LYVE-1 antibody (D). Negative control staining of the human ED with normal rabbit serum $(\mathbf{G})$. Bar $50 \mu \mathrm{m}$. ED lumen of the endolymphatic duct, $C T$ interstitial connective tissue, $B$ bone, $E P$ epithelium, $L$ lymph vessel, $D$ dermis, $V$ vein of the $V A$.

the available resolution. The fibroblast marker expressing cells were seen preferentially close to the epithelium, whereas the majority of the podoplaninexpressing cells were found more peripheral in the VA. In the mid- and distal parts of the ED, the fibroblast marker positive cells were exclusively found juxta-positioned to the basal lamina of the ED epithelium, forming a thin cell layer. Interestingly, an enrichment of these cells was observed where the epithelium was folded (Fig. 4B and C). This finding was seen on three different ED specimens.

\section{Cultured human explant ED/ES cells expressed fibroblast markers}

To explore potential functions of the human periductal CT cells, explant cultures from five different patients were established. The culturing conditions 


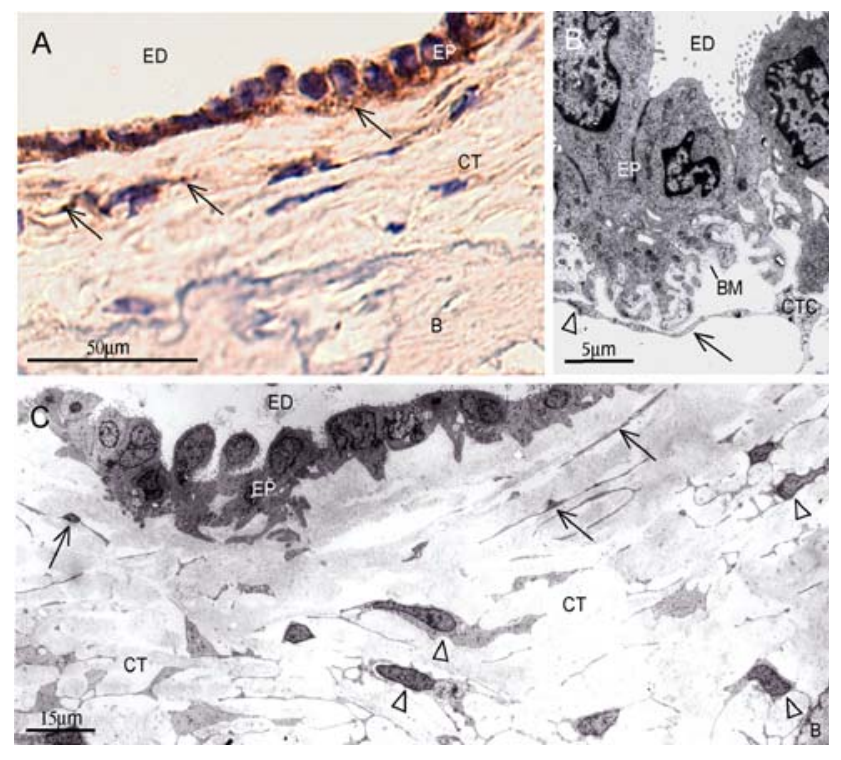

FIG. 3. A Immunohistochemical staining of the human $E D$ (106) with the anti-platelet derived growth factor receptor $\beta$ (PDGFR-B2) antibody, showing vesicular staining of $C T$ cells adjacent to the $E D$ epithelium (arrows). Bar $50 \mu \mathrm{m}$. B TEM of the human ED epithelium showing epithelia juxta-positioned $C T$ cells. Note long cellular extension in close contact with the basal lamina (arrow) and intercellular contact (arrowhead). Bar $5 \mu \mathrm{m}$. C TEM of human ED specimen indicating the presence of a thin lamellar cell type with long cellular extensions located close to the epithelium (arrows), and another more abundant large $C T$ cell type (arrowheads) with numerous cytoplasmatic extensions forming frequent intercellular contacts. Bar $15 \mu \mathrm{m}$. ED lumen of the endolymphatic duct, $C T$ interstitial connective tissue, CTC connective tissue cell, $B$ bone, EP epithelium, $B M$ basal membrane.

favored growth of fibroblast-like cells and the cultures were morphologically homogenous after four to five passages. All cell cultures were positive for Cymbus fibroblast marker, as shown by immunofluorescence staining (Table 1). Further phenotypic characterization of the ED/ES cell cultures using immunocytochemistry showed that the cells were; cytokeratin negative, $\alpha$ and $\gamma$-actin negative and smooth muscle myosin negative, calponin positive $(50 \%)$, vimentin positive $(50 \%)$ and prolyl-4-hydroxylase (fibroblast, 5B5) positive (Table 1). In conclusion, the cultured ED/ES cells were non-epithelial non-smooth muscle, actively collagen producing fibroblasts. The same staining pattern was obtained with human foreskin fibroblasts Ag 1518 (Table 1). The anti-podoplanin antibody displayed a weak but homogenous staining of both cultured ED/ES fibroblasts and Ag 1518-fibroblasts (Table 1). Immunoblotting of total lysates from the explant ED/ES cell cultures showed a variable expression of podoplanin. Two of the cell lines displayed a strong podoplaninexpression, but the expression varied with time in culture (data not shown). Human dermal fibroblasts and Ag 1518-fibroblasts showed a low expression of podoplanin.

The explant ED/ES fibroblasts compacted three-dimensional collagen gels and responded to stimulation by PDGF-BB and PGE1

In the collagen gel contraction assay described under methods, the Ag 1518-fibroblasts reduced the initial gel area to about $15 \%$ in $8 \mathrm{~h}$ (Fig. 5B). This process was promoted by platelet derived growth factor- $\mathrm{BB}$ (PDGF-BB) and inhibited by prostaglandin E1 (PGE1) (Fig. 5B), as shown earlier (Berg et al. 1998). PDGF-BB and PGE1 are CT modulating factors with opposing effects in collagen gel contraction in vitro and interstitial fluid pressure regulation in vivo.

We tested three different explant ED/ES cell lines in the collagen gel contraction assay in order to evaluate their collagen matrix interacting and gel compacting properties. The explant ED/ES cell lines performed a strong compaction of the collagen gels, and the effects of PDGF-BB and PGE1 were even more evident than with Ag 1518-fibroblasts (Fig. 5A).

TABLE 1

Immunocytochemical staining for cell markers on cultured human foreskin fibroblasts Ag1518 and human explant ED/ES connective tissue cells

Cell markers Human foreskin fibroblasts Ag1518 Human explant ED/ES cells

Vimentin, clone 3B4, DAKO

Pan-cytokeratin, Sigma

Fibroblast, clone TE-7, Cymbus ${ }^{a}$

Fibroblast prolyl-4-hydroxylase $\beta$, clone 5B5, DAKO

Calponin, clone hCP, Sigma

Smooth muscle myosin, Sigma

$\alpha$ - and $\gamma$-actin, clone HHF, DAKO

Podoplanin

$\begin{array}{cc}++ & ++ \\ 0 & 0 \\ +++ & +++ \\ +++ & +++ \\ ++ & ++ \\ 0 & 0 \\ 0 & 0 \\ (+) & (+)\end{array}$

Three different ED/ES explant cell lines were used and showed qualitatively similar staining pattern. 0 , no positive cells; $(+)$, weak staining;,$++>50 \%$ positive; +++ , all positive.

a Expression detected by immunofluorescence. 

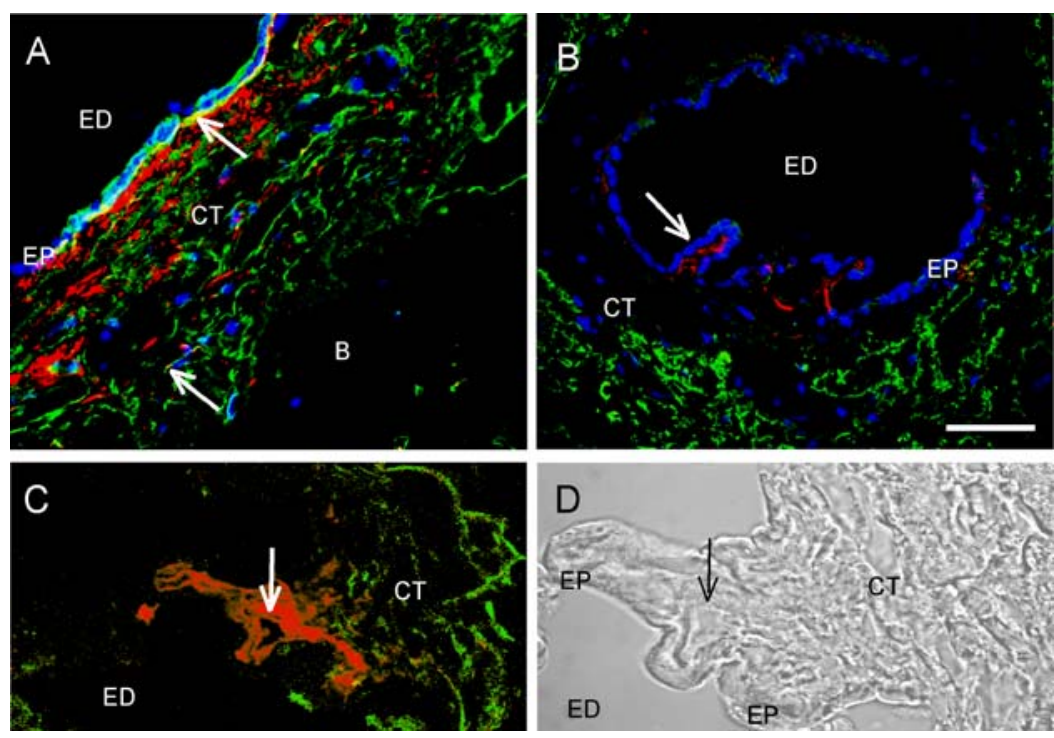

FIG. 4. Double immunofluorescence staining of the human ED106 specimen with the Cymbus anti-fibroblast antibody (red) and antipodoplanin antibody (green) in the proximal (A) and mid part (B). Arrow shows overlapping area in (A) and villous structure in (B). An enrichment of Cymbus positive cells in a villous structure of the $E D$ epithelium is shown in $(\mathbf{C})$, representing a double immuno-

Each experiment was performed in sixplicates and repeated three times. All three different cell cultures showed similar results.

\section{DISCUSSION}

In this work, we show that the interstitial CT encompassing the human ED is populated by two distinct cell phenotypes: the network-forming podo- fluorescence staining of the ED with the Cymbus anti-fibroblast antibody (red) and anti-podoplanin antibody (green) (C) and transmitted light picture of (C) (D). Arrows show the central region of a villous structure. Bar $50 \mu \mathrm{m}$. ED lumen of the endolymphatic duct, $C T$ interstitial connective tissue, $B$ bone, EP epithelium.

planin-expressing cells, and the epithelia juxta-positioned cells expressing an antigen recognized by the Cymbus fibroblast antibody.

The podoplanin-expressing cells are presumably activated CT cells rather than lymph endothelial cells. This notion is based on that the podoplanin-expressing cells did not form regular lymphatic vessels and were negative for other lymphatic endothelial markers. In a previous report (Hultgard-Ekwall et al. 2003), we showed that the network-forming periduc-

\section{A Human ED/ES fibroblasts B Human foreskin fibroblasts}

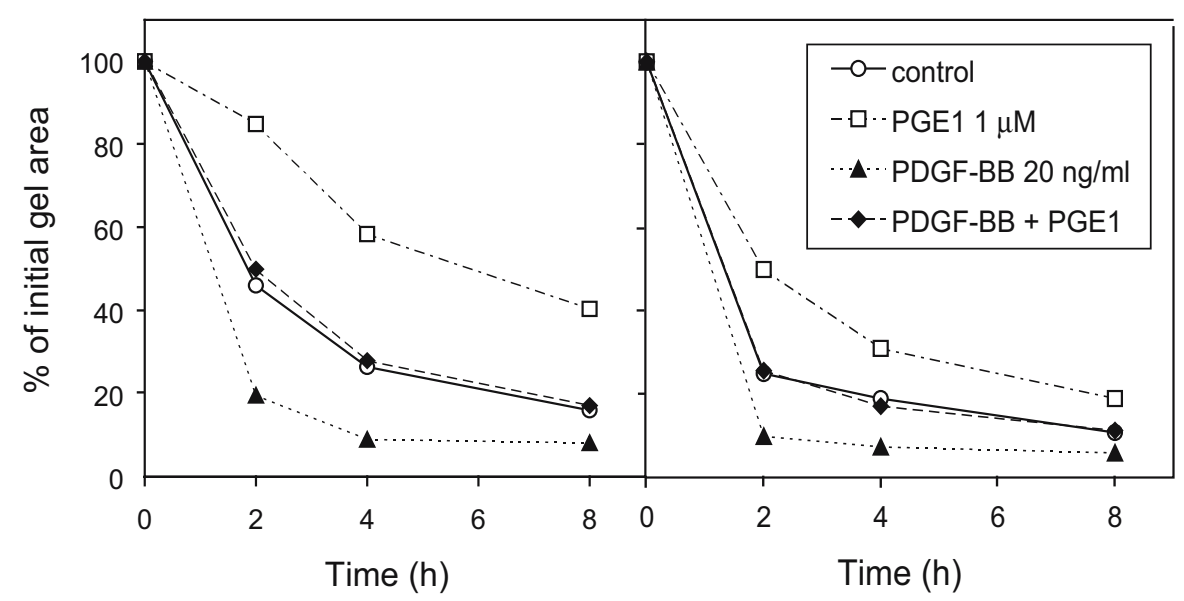

FIG. 5. Collagen gel contraction assay with human explant fibroblasts from the ED/ES (A) and human foreskin fibroblasts (Ag 1518) (B), showing the reduction in gel area at different time points with unstimulated cells (-०-), cells stimulated with prostaglandin E1 $1 \mu \mathrm{M}(-\square-)$, platelet derived growth factor-BB $20 \mathrm{ng} / \mathrm{ml}\left(-\boldsymbol{\Delta}_{-}\right)$or both $(--)$. Each value represents a mean from six individual gels. 
tal CT cells are positive for the anti-prolyl 4-hydroxylase $\beta$-subunit (5B5) antibody, which is commonly used to identify actively collagen-producing cells. We also showed that the CT cells are non-smooth muscle and non-blood endothelial cells. Furthermore, podoplanin/PA2.26 has been reported to be upregulated in vivo on murine dermal fibroblast-like cells in activated wounded skin, on cultured fibroblasts (Gandarillas et al. 1997) and on a mouse osteoblastic cell line (Nose et al. 1990). In the ED specimens, we found podoplanin-expressing CT cells in bone cavities surrounding the VA and juxta-positioned to the margin of the bone matrix. Whether these cells represent bone-forming osteoblasts, remains to be investigated.

Podoplanin is a membrane bound glycoprotein shown to be important for the formation of cell surface protrusions and to promote migration and adhesion (Schacht et al. 2003; Martin-Villar et al. 2005). In dermis podoplanin seems to be induced on interstitial CT cells during inflammation and carcinogenesis (Gandarillas et al. 1997). Interestingly, ectopic expression of PA2.26/podoplanin in mouse keratinocytes induces an epithelial-fibroblastoid morphological conversion or trans-differentiation to a phenotype with increased plasma membrane projections and enhanced motility (Scholl et al. 1999). The expression of podoplanin by CT cells, and not only by epithelia or by lymph endothelia, during normal homeostasis distinguish the periductal tissue from other investigated tissues.

The periductal and perisaccular CT cells of the inner ear form long cell surface extensions that inter connect with adjacent CT cells and with ECM components to build a tissue network (HultgardEkwall et al. 2003). It is possible that the podoplanin glycoprotein, in this work shown to be present on the periductal CT cells, by promoting adhesion or formation of cell surface protrusions is important for the formation and function of this interstitial tissue network. Interstitial CT cells in dermis and trachea actively modulate interstitial fluid pressure via forces generated by cell-ECM interactions (Reed et al. 1992; Rodt et al. 1996) and the actin cytoskeleton (Berg et al. 2001; Bronstad et al. 2002). This cellular contractility restricts the intrinsic swelling tendency of the glycosaminoglycan-rich ground substance (Wiig et al. 2003). Accordingly, it is plausible that cell-ECM contacts of periductal CT cells are important for the control of interstitial fluid pressure in the inner ear.

The mechanisms behind endolymph resorption in the inner ear are largely unknown. It is generally believed that fluid is absorbed from the ED and ES over the epithelium, convected through the surrounding interstitial CT into veins of the VA or drained directly into the sigmoid sinus intracranially (Rask-Andersen et al. 1999; Salt and DeMott 2000). We have inferred that the interstitial CT network encompassing the ED and ES, with its plausible pressure controlling properties is important for the convection of fluid through the CT. It is conceivable that the periductal CT cells by active compaction of the periductal CT facilitate the fluid fluxes over the epithelium.

The ES with its system of tubules and large epithelial surface area probably has the greatest capacity of fluid resorption. The ED in the VA constitutes a critical bottleneck for the flow of endolymph to the ES. It is likely that different mechanisms are operating to ensure and to modulate the longitudinal flow of endolymph in the duct. In the proximal part of the ED, the fibroblast marker positive cell type was abundant. In the mid- and distal parts, the fibroblast marker-expressing cells were restricted to the epithelial-mesenchymal interface and enriched under folded epithelia. Our TEM studies showed that there is a thin layer of periductal CT cells juxta-positioned to the ED epithelium. These cells formed prominent contacts with the basal lamina as well as intercellular contacts. We suggest that these cells by organizing and dragging the basal lamina and the epithelium could form involutions or villous structures of the epithelium. Hence, when the inner ear fluid pressure is high, the ED lumen could expand if the villi-forming activities of the subepithelial fibroblasts are inhibited and the periductal CT is compacted. The organizing activity of subepithelial fibroblasts might also account for the structure of the ES, recently described to be built from multiple parallel ducts or tubules rather than one sac (Hebbar et al. 1991; Linthicum and Galey 1981).

Isolated and cultured Cymbus positive fibroblasts from the human ED/ES specimens shared the same pattern of protein marker expression as cultured dermal fibroblasts (Table 1). A low podoplaninexpression was detected by immunocytochemistry on cultured primary ED/ES cell lines and on primary dermal- and Ag 1518-fibroblasts using the antipodoplanin antibody. As discussed above, fibroblasts in culture reportedly express podoplanin as a result of adopting to cell culture. It is possible that the ED/ ES cell lines also upregulate podoplanin in culture. Two of the cultured primary ED/ES cell lines displayed a strong but variable podoplanin-expression in immunoblotting experiments (data not shown). They may represent cells from the podoplanin-expressing CT cell population in the human ED.

In the collagen gel contraction assay, fibroblasts migrate and interact with each other and with the collagen fibers to organize and compact the gel (Grinnell 2003). Cultured human dermal fibroblasts 
will compact a gel to $10 \%$ of the initial gel area in 12$24 \mathrm{~h}$. The process of collagen gel compaction depends on $\beta 1$-integrins (Gullberg et al. 1990) and on intercellular adhesions and Gap-junctions synchronizing the work (Ehrlich et al. 2000). Several studies from our laboratory have shown that the characteristics of the collagen gel compaction mimic the cellular control of interstitial fluid pressure in vivo (Wiig et al. 2003; Ahlen et al. 1998; Berg et al. 1998; Heuchel et al. 1999). We found that the human explant ED/ES fibroblasts compacted collagen gels to the same degree and with the same kinetics as dermal fibroblasts. The compaction by ED/ES cells was stimulated by PDGF-BB and inhibited by prostaglandin E1. This finding demonstrates the expression of receptors for these factors on the ED/ES fibroblasts.

PDGF-BB is known to promote migration, adhesion and to restitute normal interstitial fluid pressure after anaphylactic reactions (Heldin and Westermark 1999; Heuchel et al. 1999; Rodt et al. 1996). We show in this paper that the subepithelial CT cells in the human ED express activated PDGF- $\beta$ receptors. Accordingly, it is possible that these cells participate in the control of tissue contractility and fluid content in the inner ear by a mechanism involving PDGF.

Our data points to important functions of the interstitial CT encompassing the human ED in modulating inner ear fluid pressure and endolymph resorption. Exploring the mechanisms behind these processes may lead to a better understanding of the pathophysiology of endolymphatic hydrops and Ménière's disease.

\section{ACKNOWLEDGMENTS}

We great fully acknowledge Prof. Dr. Dontscho Kerjaschki (Clinical Institute of Pathology, Medical University of Vienna, Austria) for valuable comments on the manuscript and for donating the anti-podoplanin antibody and Dr. Nikolaus Wick and Sigurd Krieger for valuable comments concerning the podoplanin stainings. We also acknowledge Marja Boström and Harald Niederegger for technical assistance. PDGF-BB was a generous gift from Prof. Carl-Henrik Heldin (Ludwig Institute for Cancer Research, Uppsala, Sweden). The work was supported by grants from The Swedish Society for Medical Research (to A.H-E), The H. and G Ankarstrand Foundation (to A.H-E), The Lennander Foundation (to A.H-E), The L. and R. Åkerham Foundation (to A.H-E), The Swedish Cancer Foundation (to K.R) and grant VR 0398, The Tysta Skolan Foundation, Sweden (to H. R-A).

\section{REFERENCES}

Ahlen K, Berg A, Stiger F, Tengholm A, Siegbahn A, Gylfe E, Reed RK, RuBIN K. Cell interactions with collagen matrices in vivo and in vitro depend on phosphatidylinositol 3-kinase and free cytoplasmic calcium. Cell Adhes. Commun. 5(6):461-473, 1998.

Berg A, Ekwall AK, Rubin K, Stjernschantz J, Reed RK. Effect of PGE1, PGI2, and PGF2 alpha analogs on collagen gel compaction in vitro and interstitial pressure in vivo. Am. J. Physiol. 274(2 Pt 2):H663-H671, 1998.

Berg A, Rubin K, Reed RK. Cytochalasin D induces edema formation and lowering of interstitial fluid pressure in rat dermis. Am. J. Physiol, Heart Circ. Physiol. 281(1):H7-H13, 2001.

Boucherot A, Schreiber R, Pavenstadt H, Kunzelmann K. Cloning and expression of the mouse glomerular podoplanin homologue gp38P. Nephrol. Dial. Transplant. 17(6):978-984, 2002.

Breiteneder-Geleff S, Matsui K, Soleiman A, Meraner P, Poczewski H, Kalt R, Schaffner G, Kerjaschki D. Podoplanin, novel 43-kd membrane protein of glomerular epithelial cells, is down-regulated in puromycin nephrosis. Am. J. Pathol. 151(4): 1141-1152, 1997.

Breiteneder-Geleff S, Soleiman A, Kowalski H, Horvat R, Amann G, Kriehuber E, Diem K, Weninger W, Tschachler E, Alitalo K, KerJaschKi D. Angiosarcomas express mixed endothelial phenotypes of blood and lymphatic capillaries: podoplanin as a specific marker for lymphatic endothelium. Am. J. Pathol. 154(2):385394, 1999.

Bronstad A, Reith A, Berg A, Reed RK. Effect of the cytoskeletal fixation agent phalloidin on transcapillary albumin transport and interstitial fluid pressure in anaphylaxis in the wistar rat. Microcirculation 9(3):197-205, 2002.

Clark RA, Folkvord JM, Hart CE, Murray MJ, McPherson JM. Platelet isoforms of platelet-derived growth factor stimulate fibroblasts to contract collagen matrices. J. Clin. Invest. 84(3): 1036-1040, 1989.

Ehrlich HP, Gabbiani G, Meda P. Cell coupling modulates the contraction of fibroblast-populated collagen lattices. J. Cell Physiol. 184(1):86-92, 2000.

Friberg U, Rask-Andersen H, Bagger-Sjoback D. Human endolymphatic duct. An ultrastructural study. Arch. Otolaryngol. 110(7): 421-428, 1984.

Gandarillas A, Scholl FG, Benito N, Gamallo C, Quintanilla M. Induction of pa2.26, a cell-surface antigen expressed by active fibroblasts, in mouse epidermal keratinocytes during carcinogenesis. Mol. Carcinog. 20(1):10-18, 1997.

Grinnell F. Fibroblast biology in three-dimensional collagen matrices. Trends Cell Biol. 13(5):264-269, 2003.

Gullberg D, Tingstrom A, Thuresson AC, Olsson L, Terracio L, Borg TK, Rubin K. Beta 1 integrin-mediated collagen gel contraction is stimulated by pdgf. Exp. Cell Res. 186(2):264$272,1990$.

Hebbar GK, Rask-Andersen, H, Linthicum, FH, Jr. Three-dimensional analysis of 61 human endolymphatic ducts and sacs in ears with and without meniere's disease. Ann. Otol. Rhinol. Laryngol. 100(3):219-225, 1991.

Heldin CH, Westermark B. Mechanism of action and in vivo role of platelet-derived growth factor. Physiol. Rev. 79(4):1283-1316, 1999.

Heuchel R, Berg A, Tallquist M, Ahlen K, Reed RK, Rubin K, Claesson-Welsh L, Heldin CH, Soriano P. Platelet-derived growth factor beta receptor regulates interstitial fluid homeostasis through phosphatidylinositol-3' kinase signaling. Proc. Natl. Acad. Sci. USA 96(20):11410-11415, 1999. 
Hultgard-Ekwall AK, Couloigner V, Rubin K, Rask-Andersen H. Network organization of interstitial connective tissue cells in the human endolymphatic duct. J. Histochem. Cytochem. 51(11): 1491-1500, 2003.

Kriehuber E, Breiteneder-Geleff S, Groeger M, Soleiman A, Schoppmann SF, Stingl G, Kerjaschis D, Maurer D. Isolation and characterization of dermal lymphatic and blood endothelial cells reveal stable and functionally specialized cell lineages. J. Exp. Med. 194(6):797-808, 2001.

Linthicum FH, JR, Galey FR. Computer-aided reconstruction of the endolymphatic sac. Acta Otolaryngol. 91(5-6):423-429, 1981.

Martin-Villar E, Scholl FG, Gamallo C, Yurrita MM, MunozGuerra M, Cruces J, Quintanilla M. Characterization of human pa2.26 antigen (tlalpha-2, podoplanin), a small membrane mucin induced in oral squamous cell carcinomas. Int. J. Cancer 113(6):899-910, 2005.

Matsui K, Breitender-Geleff S, Soleiman A, Kowalski H, Kerjaschki D. Podoplanin, a novel 43-kda membrane protein, controls the shape of podocytes. Nephrol. Dial. Transplant. 14(Suppl 1): 9-11, 1999.

Nose K, SAIto H, Kuroki T. Isolation of a gene sequence induced later by tumor-promoting 12-o-tetradecanoylphorbol-13-acetate in mouse osteoblastic cells (mc3t3-e1) and expressed constitutively in ras-transformed cells. Cell Growth Differ. 1(11):511518,1990

Rask-Andersen H, DeMott JE, Bagger-SJoback D, Salt AN. Morphological changes of the endolymphatic sac induced by microinjection of artificial endolymph into the cochlea. Hear Res. 138(1-2):81-90, 1999.

Reed RK, Rubin K, WiIG H, Rodt SA. Blockade of beta 1-integrins in skin causes edema through lowering of interstitial fluid pressure. Circ. Res. 71(4):978-983, 1992.

Rishi AK, Joyce-Brady M, Fisher J, Dobbs LG, Floros J, VanderSpeK J, Brody JS, Williams MC. Cloning, characterization, and development expression of a rat lung alveolar type i cell gene in embryonic endodermal and neural derivatives. Dev. Biol. 167(1):294-306, 1995.

Rodt SA, Ahlen K, Berg A, Rubin K, Reed RK. A novel physiological function for platelet-derived growth factor-bb in rat dermis. J. Physiol. 495 (Pt 1):193-200, 1996.

SAlt AN, DeMotT JE. Ionic and potential changes of the endolymphatic sac induced by endolymph volume changes. Hear Res. 149(1-2):46-54, 2000.

Schacht V, Ramirez Mi, Hong YK, Hirakawa S, Feng D, Harvey N, Williams M, Dvorak AM, Dvorak HF, Oliver G, Detmar M. Tlalpha/podoplanin deficiency disrupts normal lymphatic vasculature formation and causes lymphedema. EMBO J. 22(14):3546-3556, 2003.

Scholl FG, Gamallo C, Vilaró S, Quintanilla, M. Identification of pa2.26 antigen as a novel cell-surface mucin-type glycoprotein that induces plasma membrane extensions and increased motility in keratinocytes. J. Cell Sci. 112 (Pt 24): 4601-4613, 1999.

Schoppmann SF, Birner P, Studer P, Breiteneder-GelefF S. Lymphatic microvessel density and lymphovascular invasion assessed by anti-podoplanin immunostaining in human breast cancer. Anticancer Res. 21(4A):2351-2355, 2001

Wetterwald A, Hoffstetter W, Cecchini MG, Lanske B, Wagner C, Fleisch H, Atkinson M. Characterization and cloning of the e11 antigen, a marker expressed by rat osteoblasts and osteocytes. Bone 18(2):125-132, 1996.

WiIg H, Rubin K, ReED RK. New and active role of the interstitium in control of interstitial fluid pressure: potential therapeutic consequences. Acta Anaesthesiol. Scand. 47(2):111-121, 2003.

Williams MC, Cao Y, Hinds A, Rishi AK, Wetterwald A. T1 alpha protein is developmentally regulated and expressed by alveolar type I cells, choroid plexus, and ciliary epithelia of adult rats. Am. J. Respir. Cell Mol. Biol. 14(6):577-585, 1996. 